\title{
Atresia biliar perinatal (colangiopatía obliterante inflamatoria progresiva) no corregible tipo III
}

\author{
Perinatal biliary atresia (progressive, obliterative \\ cholangitis), non correctable type IIII
}

Celso Tomás Corcuera-Delgado, ${ }^{1}$ Cecilia Ridaura-Sanz, ${ }^{1}$ Flora Elba Zárate-Mondragón ${ }^{2}$

\section{RESUMEN CLÍNICO}

Niño con antecedente de ictericia desde el nacimiento, que al alta hospitalaria le indicaron baños de sol sin mostrar mejoría; los padres negaron acolia y refirieron coluria ocasional. Las pruebas de sangre reportaron elevada concentración de bilirrubinas. Con estos datos lo transfirieron al Instituto Nacional de Pediatría, a la edad de 1 mes y 23 días (primer ingreso). Durante el examen físico se comprobó la ictericia de piel, mucosas y conjuntivas; el hígado se percibía blando, con percusión total de $7 \mathrm{~cm}$. Las pruebas de función hepática resultaron con: bilirrubina total $21.04 \mathrm{mg} / \mathrm{dL}$, bilirrubina directa $13.49 \mathrm{mg} /$ $\mathrm{dL}$, bilirrubina indirecta $7.55 \mathrm{mg} / \mathrm{dL}$ (proteínas totales $5.63 \mathrm{mg} / \mathrm{dL}$, albúmina 3.06 mg/dL, globulinas 2.57 mg/dL, AST 271 U/L, ALT 195 U/L, GGT 208 U/L, fosfatasa alcalina 546 U/L, DHL 295 U/L, proteína $C$ reactiva $0.3 \mathrm{mg} / \mathrm{dL}$ ).

Se estableció el diagnóstico de síndrome colestásico neonatal prolongado con perfil TORCH negativo y tamiz metabólico ampliado normal.

En el ultrasonido abdominal se comprobó hepatomegalia y evidenció una vesícula biliar esclero-atrófica. La determinación de $\alpha-1$ antitripsina reportó 79 mg/dL (normal más de 100 mg/dL), por lo que se sospechó deficiencia.

A los tres meses de edad se registró el segundo ingreso hospitalario, donde se obtuvo una biopsia hepática percutánea, con incremento de las enzimas inflamatorias y colestasis. La biopsia no mostró datos histológicos concluyentes de obstrucción extrahepática.

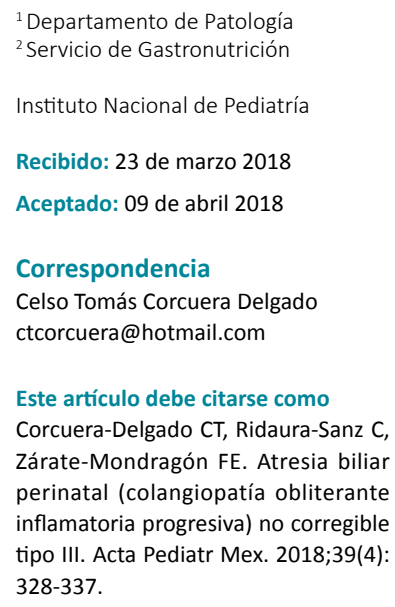

Este artículo debe citarse como Corcuera-Delgado CT, Ridaura-Sanz C, Zárate-Mondragón FE. Atresia biliar perinatal (colangiopatía obliterante inflamatoria progresiva) no corregible tipo III. Acta Pediatr Mex. 2018;39(4): 328-337. 
El paciente tuvo seguimiento en consulta externa, en el servicio de Gastronutrición cada dos meses, con incremento paulatino del hígado y aumento persistente de las enzimas inflamatorias. Se efectuó una nueva determinación de $\alpha-1$ antitripsina, que reportó concentraciones normales $(232 \mathrm{mg} / \mathrm{dL})$, al igual que las de ceruloplasmina y cobre, con lo que se descartó el diagnóstico de enfermedad de Wilson. Al no tener un diagnóstico concluyente se prescribió azatioprina y prednisona; sin embargo, no mostró mejoría, por lo que se excluyó el diagnóstico de hepatitis autoinmune.

El tercer ingreso, a los 9 meses de edad, fue por fiebre cuantificada de $38.4{ }^{\circ} \mathrm{C}$ de una semana de evolución y distensión abdominal importante, secundaria a hepatoesplenomegalia, percusión total de $15 \mathrm{~cm}$ e incremento continuo, asociada con desnutrición grave.

A los 11 meses de edad se programó para protocolo de trasplante hepático, en el Hospital Infantil de México, con diagnóstico de cirrosis criptogénica. El tratamiento médico consistió en esomeprazol, vitamina $\mathrm{K}$ y E, UDKA, rifaximina y espironolactona.

El cuarto ingreso hospitalario fue a los 15 meses de edad, debido a cuatro episodios de hematemesis (dos con coágulos y dos frescos), además de melena de dos días de evolución. Durante el examen físico se observó que el perímetro abdominal descendía, pero con red venosa colateral, telangiectasias e hígado indurado.

A los 16 meses de edad se evidenciaron várices esofágicas grado III de la clasificación japonesa y pangastropatía congestiva leve, por lo que se realizó escleroterapia de várices esofágicas.

El quinto y último ingreso al hospital se registró a los 18 meses de edad, por exantema vesicular generalizado de una semana de evolución.
El paciente tuvo fiebre de $39.1{ }^{\circ} \mathrm{C}$, melena en dos ocasiones, dermatosis polimorfa (máculas, vesículas y costras) generalizada, pruriginosa, tórax restrictivo, abdomen globoso a expensas de ascitis, con red venosa colateral, telangiectasias, hígado indurado (percusión total de $9 \mathrm{~cm}$ ) y esplenomegalia hasta la cresta ilíaca. Se inició tratamiento con vitamina $\mathrm{K}$ por tiempo prolongado, infusión de albúmina al 5\% y propanolol. Se integró el diagnóstico de hepatopatía crónica descompensada por varicela.

Al día siguiente tuvo rectorragia y hematemesis de $250 \mathrm{~mL}$. Aunque se transfundió un concentrado eritrocitario, se observó deterioro hemodinámico, somnolencia, bradicardia y dificultad respiratoria; se realizó intubación orotraqueal; durante la aspiración se obtuvieron $200 \mathrm{~mL}$ de sangre fresca. Tuvo sangrado masivo del tubo digestivo por ruptura de las várices esofágicas, que resultó en descompensación de la hepatopatía crónica por varicela. Se transfundieron dos concentrados eritrocitarios y plasma fresco congelado. Se inició tratamiento con análogos de somatostatina (octreótida) y clindamicina por broncoaspiración; sin embargo, la evolución no fue favorable y falleció.

\section{Comentario clínico}

\section{(Dra. Flora Zárate Mondragón)}

La ictericia representa un reto diagnóstico para el clínico. Al inicio debe definirse si es provocada por hiperbilirrubinemia indirecta o directa. Uno de los problemas más comunes es confundir la ictericia fisiológica o por leche materna con ictericia originada por colestasis. Por tanto, cuando el paciente manifiesta ictericia por más de dos semanas, hipocolia o acolia y coluria, deberá investigarse la causa.

En el caso aquí reportado se indicaron, inicialmente, baños de sol, pues el diagnóstico 
sugerente se asociaba con ictericia fisiológica, pero al no desaparecer se practicaron pruebas de funcionamiento hepático que resultaron alteradas; el ultrasonido no mostró atresia de vías biliares, por lo que fue enviado al Instituto Nacional de Pediatría.

A su ingreso contaba con elementos suficientes para recibir tratamiento por colestasis neonatal prolongada, que se define como la concentración de bilirrubina directa mayor de $1 \mathrm{mg} / \mathrm{dL}$ en caso de que la bilirrubina total sea de $5 \mathrm{mg} / \mathrm{dL}$ o, bien, más de $20 \%$ de la concentración total en caso de ser mayor de $5 \mathrm{mg} / \mathrm{dL}$, con inicio en los tres primeros meses del nacimiento y se prolongue por más de 15 días. Además, a la exploración física tenía ictericia y hepatomegalia.

La colestasis neonatal afecta a 1 de cada 2500 lactantes y su origen se relaciona con causas infecciosas, anormalidades anatómicas del sistema biliar, alteraciones endocrinas y genéticas, errores innatos del metabolismo, exposición a fármacos o toxinas, alteraciones vasculares, etc. (Cuadro 1). Por ello, en pacientes con colestasis debe efectuarse un análisis cauteloso, con la finalidad de establecer el diagnóstico certero. Por frecuencia y urgencia en el diagnóstico, una de las enfermedades que debe descartarse en primera instancia es la atresia de vías biliares, pues representa la causa más común de colestasis en 30-40\% de los casos. En este paciente, el cuadro clínico no reportó datos de hipocolia ni acolia; sin embargo, las pruebas de función hepática resultaron con elevadas concentraciones de ALT, AST y fosfatasa alcalina.

La gamaglutamiltranspeptidasa es un indicador de daño canalicular de la atresia de vías biliares, definida por la concentración mayor de 300 UI/L. Nuestro paciente reportó $200 \mathrm{UI} / \mathrm{L}$, cifras no compatibles con el diagnóstico clínico ni de laboratorio de atresia de vías biliares. Posteriormente se solicitó un segundo ultrasonido, como parte del protocolo diagnóstico de colestasis pro-
Cuadro 1. Diagnóstico diferencial de colestasis neonatal

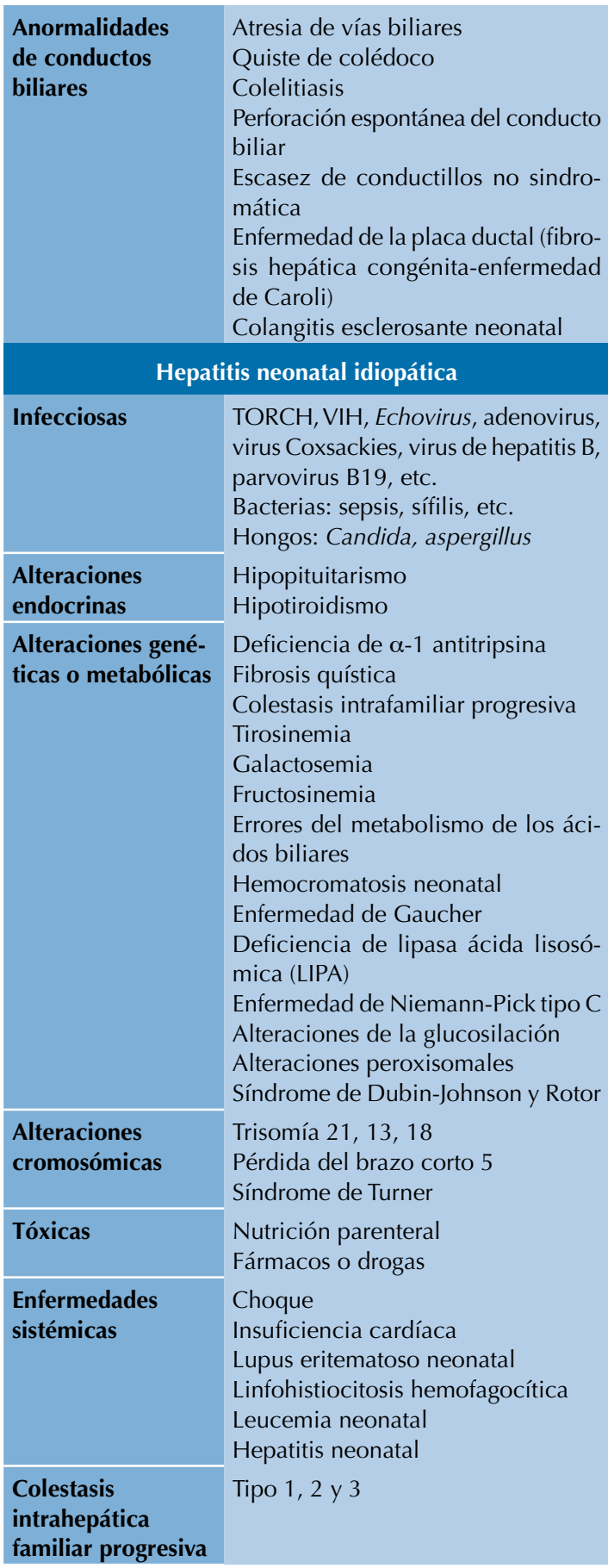

longada. Es un estudio accesible y mínimamente invasivo que aporta datos de gran relevancia para 
descartar la presencia de quiste de colédoco, hemangiomas o tumores hepáticos y dilataciones del árbol biliar; además, permite estimar el tamaño del hígado y sus características, y proporciona datos sugerentes de atresia de vías biliares, como la presencia del cono fibroso en forma triangular y, ecogénicamente, denso en la porta hepatis, en la bifurcación craneal de la vena porta. Su valor predictivo positivo es de $95 \%$, aunque este signo es dependiente del operador del estudio.

El cordón fibroso también puede estar enmascarando inflamación difusa del parénquima hepático. Nuestro paciente no reportó signos sugerentes de atresia de vías biliares. La biopsia hepática puede orientar a una obstrucción canalicular cuando están presentes marcadores típicos como: proliferación de conductos biliares, lagos y tapones que afectan todos los espacios porta; sin embargo, depende del momento en que se obtenga; por ejemplo, cuando se realiza tempranamente, antes de las 6 semanas de vida, los hallazgos distintivos pueden no estar presentes. La biopsia obtenida en el paciente de este estudio no mostró datos compatibles con obstrucción biliar. Figura 1
Cuando los hallazgos clínicos y los estudios de laboratorio y gabinete no orientan al diagnóstico de atresia de vías biliares debe establecerse el diagnóstico diferencial con otras enfermedades. Para descartar las causas infecciosas se realiza un perfil TORCH completo y se solicitan radiografías de huesos largos; cuando el paciente muestra datos de infección aguda deben tomarse cultivos microbianos. Nuestro paciente reportó negativo el perfil TORCH, las radiografías de huesos largos no mostraron alteraciones, ni se encontraron datos clínicos ni de laboratorio para sospechar un cuadro de sepsis.

Posteriormente deben descartarse los errores innatos del metabolismo y enfermedades por depósito, por ejemplo: deficiencia de $\alpha-1$ antitripsina, una enfermedad metabólica importante que requiere trasplante hepático; por tanto, es importante determinar la concentración de $\alpha-1$ antitripsina y detectar sus alelos en suero. En el caso aquí expuesto, el primer análisis reportó concentraciones por debajo de los límites de referencia, por lo que se sospechó esta enfermedad; sin embargo, la biopsia no se relacionó con este diagnóstico, si bien es cierto que la

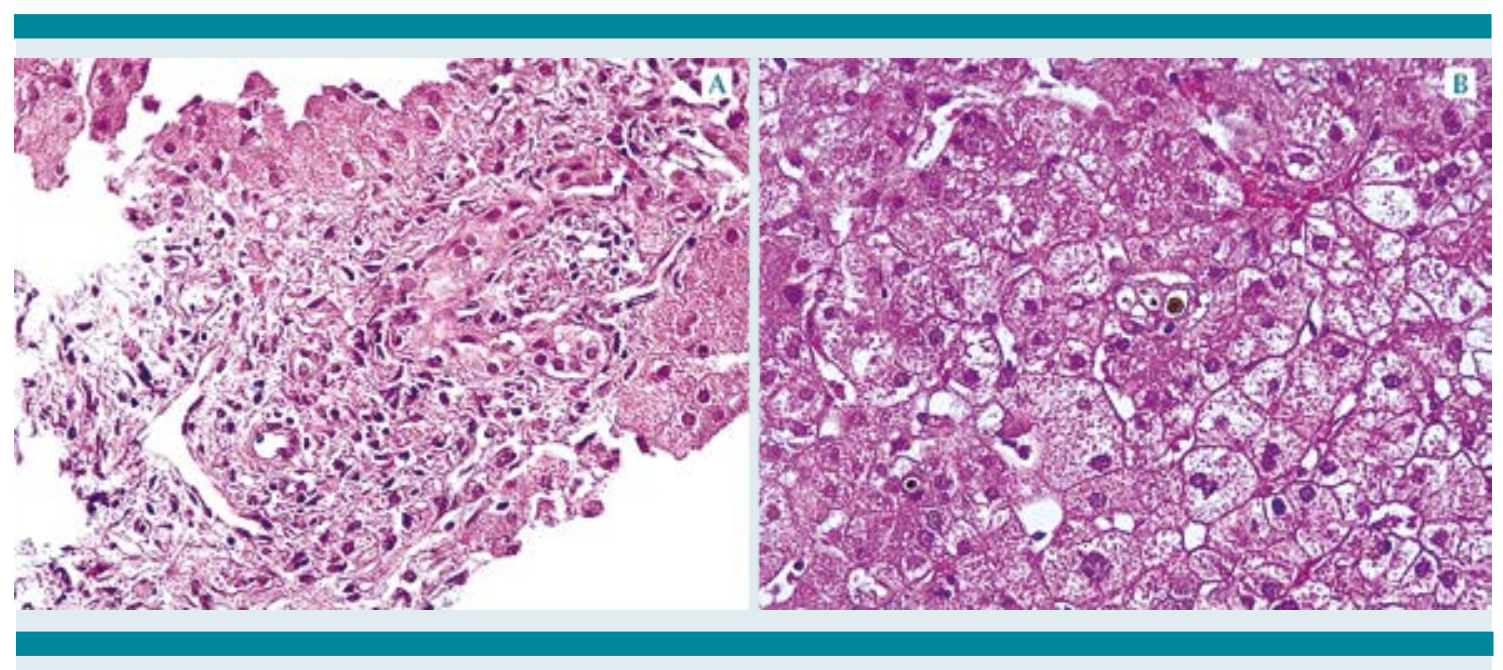

Figura 1. A: Espacio porta con infiltrado linfohistiocítico leve inespecífico, sin proliferación de conductos ni tapones biliares. B: lobulillo con colestasis intracitoplásmica e intracanalicular, y degeneración plumosa de los hepatocitos. 
deficiencia de $\alpha-1$ antitripsina puede presentar cualquier espectro de daño detectado en la biopsia hepática, en este caso no se encontraron los marcadores típicos y al repetir el estudio de laboratorio no se observaron alteraciones. La $\alpha-1$ antitripsina es una proteína reactante de fase aguda; por tanto, en pacientes con algún proceso inflamatorio hepático puede estar elevada y enmascarar la enfermedad, por lo que debe efectuarse la medición-detección de los alelos.

A nuestro paciente también se le practicó el tamiz metabólico ampliado, que permite descartar errores innatos del metabolismo de moléculas pequeñas y determina la concentración de sustancias que se acumulan en la sangre y orina, como: tirosina, fenilalanina, galactosa, fructosa, ácidos orgánicos (orina), etc.; entonces, al no detectar elevadas concentraciones de estos metabolitos, se descartan tales enfermedades. No obstante, al no establecer el diagnóstico definitivo, se estudió la posibilidad de tirosinemia, con la medición en orina y suero de succinil-acetona, pero también se descartó esta enfermedad. También se descartaron: enfermedad de Wilson (medición de ceruloplasmina y cobre en suero y orina) y deficiencia de lipasa ácida (medición enzimática). La biopsia hepática, además del diagnóstico de obstrucción, es útil para establecer el diagnóstico de enfermedades por atesoramiento (glucogenosis, Wolman, Gaucher, etc.), que también se descartaron. La biopsia de nuestro paciente sólo demostró inflamación inespecífica con datos de cirrosis. Figura 2

Otra causa de colestasis que debe considerarse es la hepatitis autoinmune; sin embargo, al no encontrar un origen específico, se prescribió tratamiento de prueba con prednisona y azatioprina, pero al no obtener reacción satisfactoria (disminución del cuadro clínico y normalización de las pruebas de funcionamiento hepático), se suspendió el tratamiento y descartó este diagnóstico.
Otra enfermedad importante en los últimos años es la colestasis intrahepática familiar progresiva, que representa un conjunto de enfermedades autosómicas recesivas que alteran la formación de la bilis; sin embargo, según el tipo, suelen detectarse concentraciones bajas o normales de GGT, con colesterol normal (datos que el paciente no tenía) y en la biopsia se observa colestasis intracanalicular y citoplasmática con daño hepatocelular, manifestado con pérdida de la arquitectura normal, con transformación gigantocelular y pseudoacinos; en estadios más avanzados hay escasez de conductos. Sin embargo, el diagnóstico se establece con estudios moleculares.

Puesto que el paciente no tenía causa específica, pero mostraba datos clínicos de cirrosis, se envió a trasplante hepático; sin embargo, esta posibilidad no se concretó y el paciente tuvo todas las complicaciones secundarias a la cirrosis: hipertensión portal (várices esofágicas, hiperesplenismo, ascitis) e inmunodeficiencia secundaria (desnutrición grave), lo que originó su deceso, por complicaciones de la infección por virus de la varicela.

La evolución clínica desfavorable, la progresión rápida a cirrosis descompensada y el ultrasonido con reporte de vesícula biliar esclero-atrófica fueron indicaciones para repetir la biopsia en busca de diagnósticos diferenciales o datos de obstrucción.

\section{Comentario anatomopatológico}

\section{(Dr. Celso Tomás Corcuera Delgado)}

Se realizó la autopsia completa. De manera externa mostraba ictericia intensa, afectación de la piel y todas las mucosas. El hígado estaba aumentado de peso y tamaño (480 vs 350 g), tenía tinte verdoso, nodularidad difusa y consistencia aumentada. No tenía la vesícula biliar; las vías 


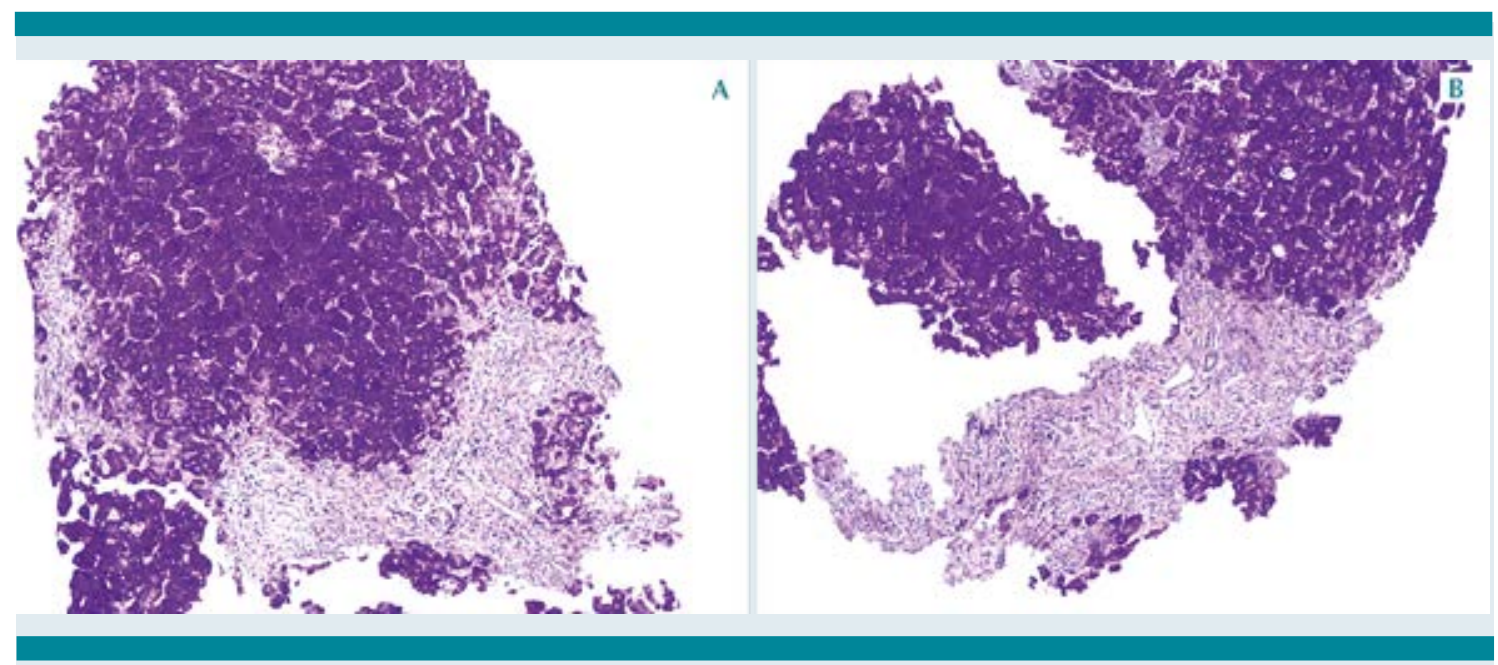

Figura 2. A y B (tinción PAS): parénquima hepático con distorsión de la arquitectura lobulillar, se delimita el parénquima hepático en nódulos irregulares y pequeños grupos de hepatocitos residuales atrapados por la fibrosis que se origina en los espacios porta, rompe la placa limitante y forma algunos puentes porto-portales.

biliares extrahepáticas se palparon de consistencia fibrosa y en los cortes seriados no se evidenció luz permeable. El bazo también estaba aumentado de tamaño y peso (150 vs 35 g). Figura 3

Los cortes histológicos adyacentes al páncreas mostraron el conducto colédoco (conducto biliar común) con obliteración parcial de la luz, debido a estenosis fibrosante. El conducto

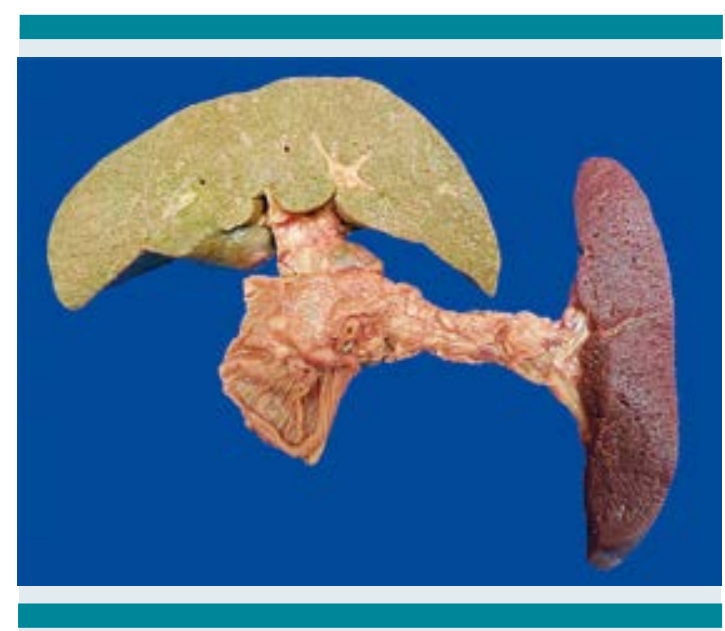

Figura 3. Hepatoesplenomegalia. Hígado verdoso con nodularidad difusa y ausencia de vesícula biliar. hepático común solo mostró un cordón fibroso sin remanentes de la vía biliar. Figura 4

Los cortes del parénquima hepático exhibían tabiques fibrosos que disecaban y rodeaban nódulos redondeados e irregulares de hepatocitos, que a diferencia de otras formas de cirrosis éstas son características de la cirrosis biliar. Los islotes parenquimatosos estaban deglutidos por más tejido fibroso y edema; no obstante, en la biopsia las bandas de tejido fibroso fueron más amplias y el hígado tenía estructura mixta micro y macronodular. Figura 5

Con estos hallazgos, el diagnóstico estuvo representado por la enfermedad principal:

I. Atresia biliar perinatal (colangiopatía obliterante inflamatoria progresiva) no corregible (tipo III )

1. Obliteración fibrosa de la vesícula biliar y de la vía biliar extra e intrahepática.

2. Historia clínica de vesícula biliar esclero-atrófica. 


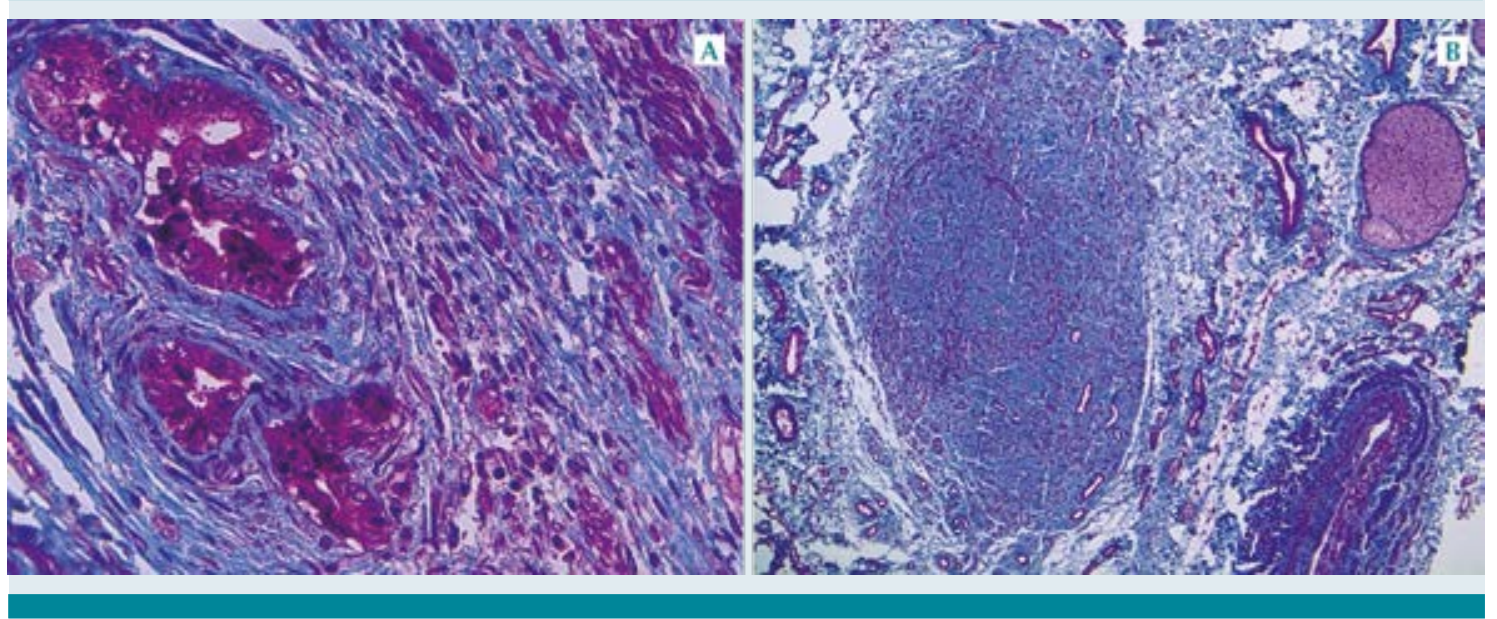

Figura 4. (Tricrómico de Masson). A: conducto colédoco con obliteración fibrosa acentuada y remanente de epitelio biliar. B: cordón fibroso del conducto hepático común.

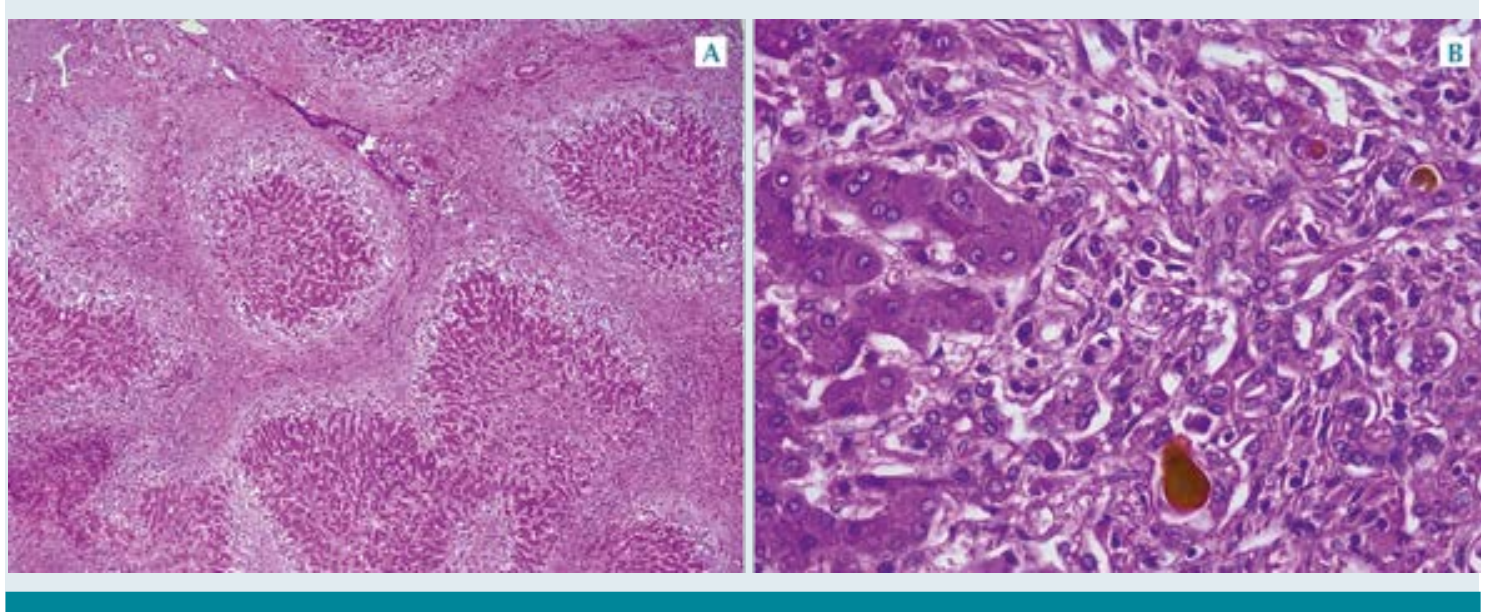

Figura 5. A (vista panorámica): cirrosis biliar. B (acercamiento): proliferación de conductos portales y neocolangiolos con tapones de bilis en la luz.

II. Cirrosis biliar obstructiva micro y macronodular secundaria

Se encontraron complicaciones propias de la atresia biliar, representadas por:

III. Datos anatómicos de insuficiencia hepática

1. Ictericia generalizada.
2. Colestasis multivisceral (hígado, glándulas suprarrenales, riñón, ganglios linfáticos, bazo y médula ósea).

3. Encefalopatía hepática.

a. Degeneración astrocítica de Alzheimer tipo II

b. Historia clínica de hiperamonemia

4. Edema escrotal 
IV. Datos anatómicos de hipertensión portal

1. Ascitis de $350 \mathrm{~mL}$

2. Circulación colateral

3. Várices gastro-esofágicas

4. Esplenomegalia congestiva (150g vs 35g)

V. Pancreatitis biliar intersticial aguda leve y multifocal

Como enfermedades concomitantes se establecieron:

VI. Desnutrición moderada estimada por el índice Kanawati-McLaren

VIII. Infección por varicela con necrosis hepática multifocal y nefritis intersticial

\section{DISCUSIÓN}

(Dra. Cecilia Ridaura Sanz)

La atresia de vías biliares se define como una colangiopatía inflamatoria fibrosante que afecta el árbol biliar extra-hepático, lo que resulta en destrucción y obstrucción del conducto biliar. El proceso obliterante varía en cuanto al tiempo de evolución y puede afectar diferentes segmentos del árbol biliar. El fenotipo de atresia de vías biliares puede representar el proceso final común de diversas enfermedades..$^{1-3}$ Se reconocen dos formas de atresia de vías biliares: ${ }^{4}$ la más común es la forma perinatal, que representa la mayoría de los casos, donde los niños suelen tener apariencia sana y peso promedio; sin embargo, tienen antecedente de heces pigmentadas al nacimiento que evolucionan a acolia, con subsiguiente manifestación de ictericia entre las 4 y 8 semanas de edad. La forma embriónica o fetal más frecuente ocurre en $10-35 \%$ de los casos. ${ }^{4}$ Estos niños padecen acolia y colestasis desde el nacimiento, son prematuros o hipotróficos y muestran anomalías congénitas asociadas, como poliesplenia y asplenia, defectos cardiovasculares, situs inversus abdominal, malrotación intestinal y otras malformaciones de la vena porta y arteria hepática, y su pronóstico es malo. ${ }^{5}$

La forma perinatal, como la ocurrida en el paciente de este estudio, quizá fue provocada por múltiples factores; sin embargo, resalta la intervención de agentes virales o tóxicos en individuos con predisposición genética. La forma fetal es resultado de un defecto de la morfogénesis, ya sea por malformación primaria o interrupción del desarrollo en etapas tempranas de la formación de la placa biliar asociada con el síndrome de poliesplenia. ${ }^{6-8}$

La clasificación anatómica de atresia de vías biliares mejor comprendida es la del registro japonés (revisada por Ohi $)^{9}$ que incluye: tipo I, atresia del conducto colédoco ( $10 \%$ de los pacientes); tipo II, atresia del conducto hepático común ( $2 \%$ de los pacientes); $y$ tipo III, atresia de la porta hepatis (88\% de los casos). Los términos "corregible" o "no corregible" se refieren a la posibilidad de practicar una resección del segmento obliterado y anastomosis de las porciones permeables, como sucede en pacientes cuando solo se oblitera en forma segmentaria el colédoco o del conducto hepático común. En la actualidad esta clasificación ha perdido significado, debido a los procedimientos quirúrgicos de reparación del árbol biliar. En el caso que presentamos, la obliteración abarcó todo el árbol biliar, por lo que se estableció la clasificación "no corregible tipo III".

Un aspecto importante de comentar en este caso es la utilidad de la biopsia hepática para definir si existe o no obstrucción extra-hepática. Los criterios histológicos utilizados para distinguir entre hepatitis neonatal y atresia biliar, que representan las dos alteraciones más frecuentes de colestasis neonatal prolongada, se muestran en el cuadro 2.

Un estudio efectuado en el Instituto Nacional de Pediatría, en el que seleccionaron 110 niños 
Cuadro 2. Criterios histológicos para el diagnóstico diferencial entre atresia biliar y hepatitis neonatal

\begin{tabular}{|c|c|c|}
\hline Alteración & Hepatitis neonatal & Atresia de vías biliares \\
\hline Proliferación de conductos biliares interlobares & Focal e irregular & Sí, acentuada y universal \\
\hline Proliferación de colangiolos (ductular) & Focal en colestasis prolongada & Sí, universal \\
\hline Tapones biliares en conductos interlobares & No & Sí \\
\hline Edema y fibrosis portal & Variable, tardía e irregular & $\begin{array}{c}\text { Constante, temprana, progresiva } \\
\text { y universal }\end{array}$ \\
\hline Hematopoyesis & Sí & No u ocasional \\
\hline Células gigantes multinucleadas & Sí & Sí \\
\hline Alteración de la arquitectura lobulillar & Sí & En fases tardía "cirrosis biliar" \\
\hline
\end{tabular}

Fuente: Ridaura CS. Biopsia hepática. Acta Pediátrica México. 2008;29(4):216-226

con colestasis neonatal prolongada, para comparar la sensibilidad, especificidad y valores predictivos positivos de las características clínicas, concentración de bilirrubina, evaluación de gammagrafía hepática y biopsia hepática por punción reportó que esta última ofrece los mejores valores predictivos para distinguir entre enfermedad obstructiva extra-hepática versus no obstructiva. ${ }^{10}$

Este método reporta algunos resultados falsos negativos para obstrucción. Uno de ellos implica obtener la biopsia en etapas tempranas y el otro es la obtención de tejido hepático insuficiente o inadecuado, como el que se aplicó en el presente caso. La biopsia estudiada en nuestro paciente estaba fragmentada y reveló un proceso de cirrosis, establecido por la coexistencia de grandes bandas de fibrosis. Esa fibrosis provocó fragmentación del tejido, por lo que la mayor parte de los fragmentos analizados solo mostraron la parte del lobulillo sin fibrosis. En los pacientes con cirrosis biliar en etapa avanzada pueden observase zonas de fibrosis con desaparición de los conductos interlobares y, por tanto, los criterios de obstrucción no son evidentes. Como se aprecia en la Figura 2, la fibrosis no mostró conductos evaluables, lo que impidió establecer el diagnóstico definitivo en vida.
Pese a lo anterior, el pronóstico del paciente no habría cambiado, pues debido a la demora en la atención médica, la única posibilidad terapéutica pudo ser el trasplante hepático, porque se ha demostrado que la cirugía de porto-enteroanastomosis (operación de Kasai) es poco exitosa en pacientes con cirrosis biliar establecida. ${ }^{11}$

La causa de muerte del paciente obedeció a complicaciones de la enfermedad biliar; la varicela en sus últimos días pudo agravar el daño hepático, pues se encontraron datos de infección en algunos hepatocitos; sin embargo, al no ser la causa inmediata del fallecimiento, se consideró como enfermedad contribuyente.

\section{CONCLUSIÓN}

Los pacientes con atresia de vías biliares suelen diagnosticarse en etapas tardías en nuestro medio, con subsiguiente fallecimiento en forma temprana. Diversos estudios exponen que a pesar del éxito transitorio de la portoentero-anastomosis, la mayoría de los pacientes requiere trasplante hepático a largo plazo. En nuestro caso no se llevó a cabo el tratamiento quirúrgico, debido a que el diagnóstico se estableció de manera postmortem. Por lo tanto, no fue posible beneficiarlo del tratamiento vigente 
recomendado. La biopsia hepática por punción es un excelente método diagnóstico que debe interpretarse estableciendo la correlación con el contexto clínico y en caso de persistir los datos de obstrucción debe repetirse o proceder a la exploración directa de la vía biliar a través de laparotomía diagnóstica.

\section{REFERENCIAS}

1. Lee JY, Sullivan K, El Demellawy D, Nasr A. The value of preoperative liver biopsy in the diagnosis of extrahepatic biliary atresia: A systematic review and meta-analysis. J Pediatr Surg 2016;51(5):753-61. doi: 10.1016/j.jpedsurg.2016.02.016.

2. Kerkeni Y, Ksiaa A, Belghith M, Sahnoun L, Maazoun K, Krichene I, Mekki M, Abdellatif $\mathrm{N}$. Biliary atresia: experience of a nord African center. Tunis Med 2015;93(11):683-6.

3. Silveira TR, Salzano FM, Howard ER. Congenital structural abnormalities in biliary atresia: evidence for etiopathogenic heterogeneity and therapeutic implications. Acta Paediatr Scand 1991;80(12):1192-1199.

4. Balistreri WF, Bove KE, Rickman FC. Biliary atresia and other disoders of the extrahepatic bile ducts. In: Suchy
FJ, Sokol R, Balistreri WF, editors. Liver disease in children. $2^{\text {nd }}$ ed. Philadelphia: Lippincott Williams and Wilkins, 2001;253-274.

5. Vazquez J, López-Gutiérrez JC, Gamez M. Biliary atresia and the polysplenia syndrome: its impact on final outcome. J Pediatr Surg 1995;30(3):485-487.

6. Davenport M, Savage M, Mowat AP. Biliary atresia splenic malformation syndrome: an etiologic and prognostic subgroup. Surgery 1993;113(6):662-668.

7. Tanano H, Hasegawa T, Kawahara H. Biliary atresia associated with congenital structural anomalies. J Pediatrsurg 1999; 34(11):1687-1690.

8. Karrer FM, Hall RJ, Lilly JR. Biliary atresia and the polysplenia syndrome. J Pediatr Surg 1991;26(5):524-527.

9. Ohi R, Masaki N. The jaundiced infant: biliary atresia and other obstructions. In: O'Neill JA, Rowe M, Grosfeld $\mathrm{JL}$, editors. Pediatric surgery. $1^{\text {ts }}$ ed. St Louis: Mosby; 1998:1465-1482.

10. Ridaura-Sanz C, Navarro-Castilla E. Papel de la biopsia hepática en el diagnóstico de colestasis prolongada en lactantes. Rev Inv Clin 1992; 44:193-202.

11. Serinet MO, Wildhaber BE, Broué p. Impact of age of Kasai operation in its results in late childhood and adolescence: a rational basis for biliary atresia screening. Pediatrics 2009;123(5):1280-1286.

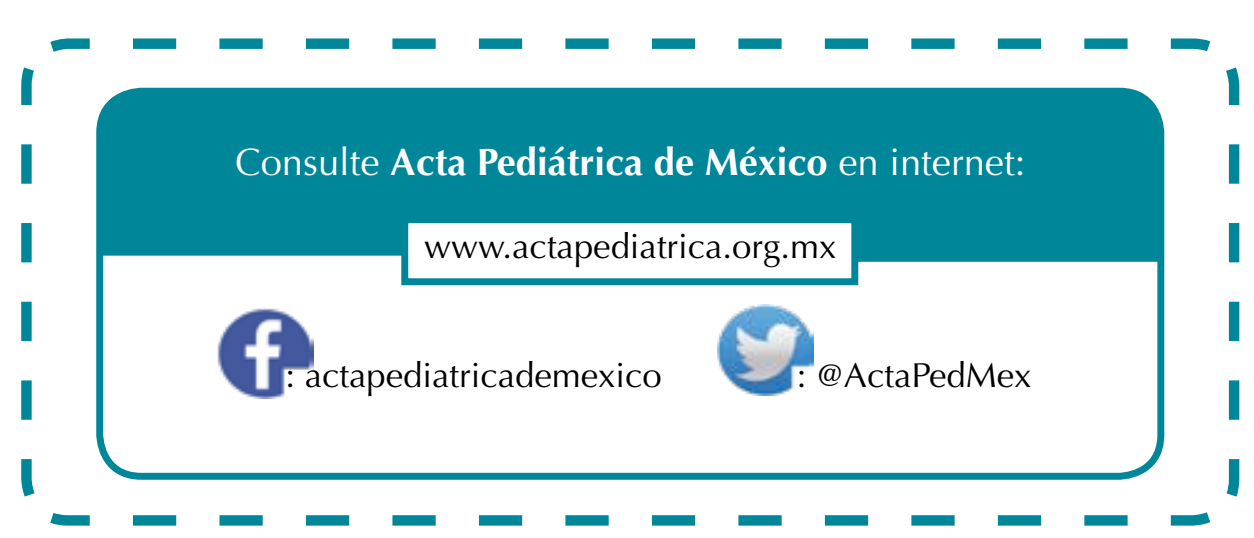

